
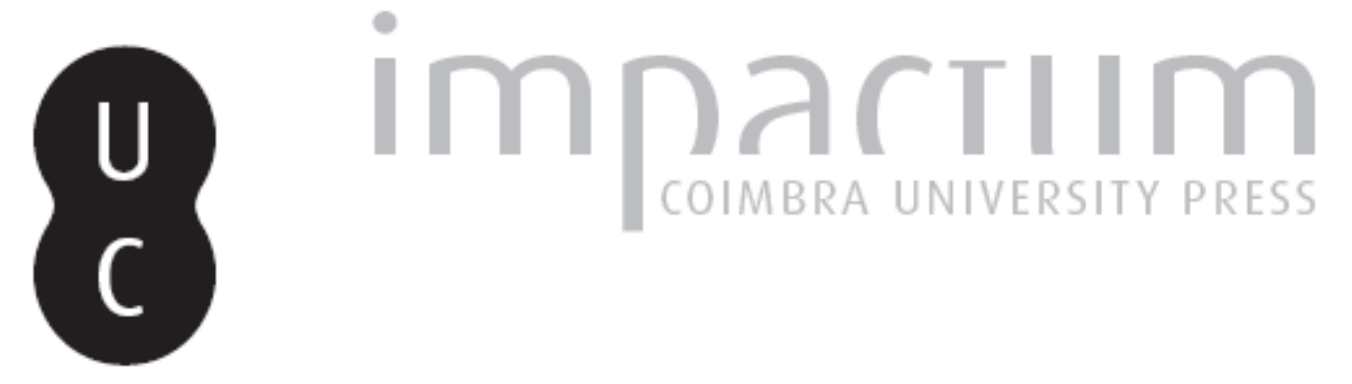

Un compte rendu de tome 4, libro V de la commentaire sur la République de Vegetti

Autor(es): Brisson, Luc

Publicado por: Imprensa da Universidade de Coimbra

URL persistente: URI:http://hdl.handle.net/10316.2/42276

DOI: DOI:https://doi.org/10.14195/2183-4105_2_8

Accessed : $\quad$ 26-Apr-2023 11:42:24

A navegação consulta e descarregamento dos títulos inseridos nas Bibliotecas Digitais UC Digitalis, UC Pombalina e UC Impactum, pressupõem a aceitação plena e sem reservas dos Termos e Condições de Uso destas Bibliotecas Digitais, disponíveis em https://digitalis.uc.pt/pt-pt/termos.

Conforme exposto nos referidos Termos e Condições de Uso, o descarregamento de títulos de acesso restrito requer uma licença válida de autorização devendo o utilizador aceder ao(s) documento(s) a partir de um endereço de IP da instituição detentora da supramencionada licença.

Ao utilizador é apenas permitido o descarregamento para uso pessoal, pelo que o emprego do(s) título(s) descarregado(s) para outro fim, designadamente comercial, carece de autorização do respetivo autor ou editor da obra.

Na medida em que todas as obras da UC Digitalis se encontram protegidas pelo Código do Direito de Autor e Direitos Conexos e demais legislação aplicável, toda a cópia, parcial ou total, deste documento, nos casos em que é legalmente admitida, deverá conter ou fazer-se acompanhar por este aviso. 


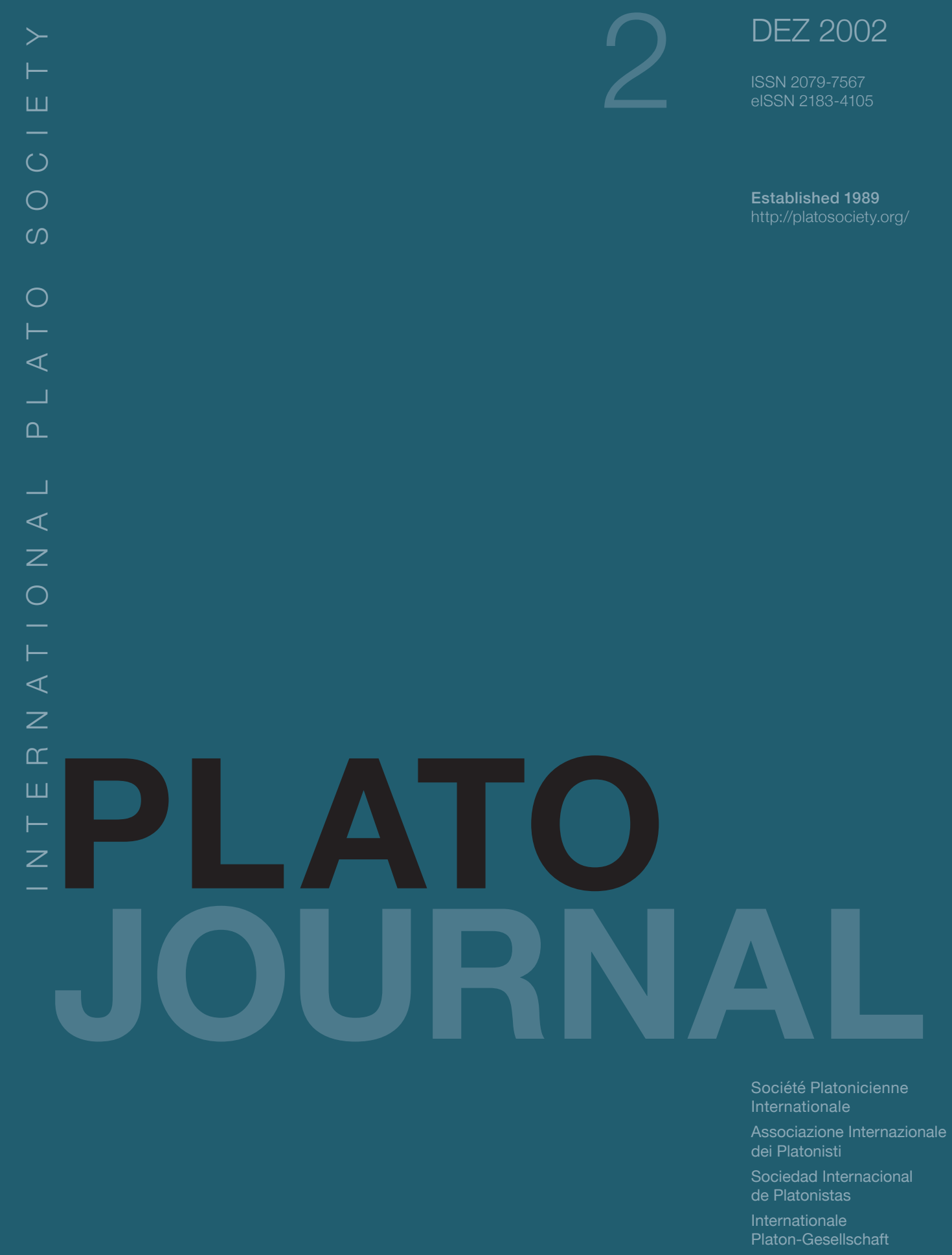




\title{
Un compte rendu de tome 4, libro $V$ de la commentaire sur la République de Vegetti
}

\author{
BRISSON, Luc, in 2. Plato 2 (2002), [En
}

ligne], January 2008

Libro V, coll. Elenchos XXVIII, 4, Napoli (Bibliopolis) 200, 563 pp.: Introduction par Mario Vegetti, 15-38; Libro V (Traduction et notes) par Mario Vegetti, 39-104. Commentaires: A. Beltista eiper dunata. Lo statuto dell'utopia nella Repubblica, par Mario Vegetti 107-47; Ba. La prima ondata: il "dramma femminile", par Silvia Campese, 149-195; Bb. Nudità, par Silvia Campese, 197-207; C. La technê antilogikê tra erizein e dialegesthai, par Fulvia de Luise et Giuseppe Farinetti, 209-31; D. L'utopia dalla commedia al dialogo platonico, par Anna Beltrametti, 233-56; E. La seconda ondata: la comunanza di donne e figli, par Silvia Campese, 257-93; F. La "razza pura", par Mario Vegetti, 295-300; G. La guerra della kallipolis, par Silvia Gastaldi, 301-34; H. Il regno filosofico, par Mario Vegetti, 335-64; I. Teoria delle idee e ontologia, par Franco Ferrari, 365-91; L. Conoscenza e opinione: il filosofo e la città, par Franco Ferrari, 393-419; Ma. Aristotele discute la Repubblica, par Francesca Calabi, 421-38; Mb. La critica aristotelica alla Repubblica nel secondo libro della Politica, il Timeo et le Leggi, par Mario Vegetti, 439-452; N. La kallipolisdi Rousseau, par Fulvia de Luise, 453-496; O. Il confronto di Marx con Platone (attraverso Hegel), par Guiseppe Farinetti, 497-559; errata corrige [vol. I-IV], 561-2.

Voici la suite de l'entreprise menée par Mario Vegetti, la traduction commenté de la République de Platon. Le texte traduit est celui établi par Burnet. La traduction est celle de Mario Vegetti qui a fait précéder sa traduction d'une introduction, et qui l'explicite par des notes en bas de page. Cette traduction est accompagnée de commentaires longs et élaborés faits par un groupe de "spécialistes" (studiosi) qui, pour la plupart, appartiennent, comme le Prof. Mario Vegetti, au Département de Philosophie de l'Université de Pavie. J'ai déjà rendu compte des trois premiers tomes de ce commentaire qui portaient sur les quatre premiers livres de la République. Je présente ici le quatrième tome, qui a pour objet le cinquième livre de ce dialogue platonicien.

C'est à l'occasion d'une comparaison entre la justice dans l'individu et dans la cité que, au début du livre II de la République, la constitution politique est prise pour objet. Très rapidement la notion d'"individu" est remplacée par celle d'"âme". Voilà pourquoi on peut, comme l'a fait Jean-François Pradeau (Platon et la cité, coll. Philosophie, Paris (PUF) 1997, parler de "psychologie politique" en ce qui concerne la République. Les livres II à IV exposent la constitution de la cité, de telle sorte que la Républiqueaurait pu s'arrêter là. Les livres V à VII sont consacrés aux trois vagues d'objections qui pourraient engloutir le projet: exiger des femmes qu'elles partagent toutes les tâches, y compris celle de la défense; instituer la communauté des femmes et des enfants; donner le pouvoir aux philosophes. Et enfin, les livres VIII à X décrivent le devenir, c'est-à-dire la corruption de la constitution excellente, avant de revenir au point de départ en définissant l'homme juste.

Le cinquième livre de la République, qui est ici traduit et commenté, constitue une provocation à l'endroit de la démocratie athénienne, car les conditions de réalisation de la cité juste sont autant de remises en cause de la démocratie athénienne. Exiger que les femmes participent à la défense de la cité (V 451c-457b), c'est leur reconnaître un statut qui leur était refusé. En Grèce ancienne et notamment à Athènes en effet, la femme se définit essentiellement comme épouse et mère de famille; elle occupe le domaine privé et elle se trouve exclue du domaine public et donc de la citoyenneté. Par ailleurs, instituer la communauté des femmes et des enfants (V 457c-471c), c'est saper l'un des fondements de la démocratie athénienne, établie sur un partage du territoire et des richesses qui avaient pour base la propriété domestique (dans l'ô̂kos). Cela dit, il est plus difficile de déterminer si la remise du pouvoir au philosophe (V 471c- VI 541b) était considérée par les contemporains de Platon comme une provocation ou tout simplement comme le rêve futile d'un savant.

Je me contenterai ici de proposer un bref résumé de chaque section du commentaire à ce cinquième livre. Je sollicite, d'entrée de jeu, la bienveillance du lecteur et surtout l'indulgence des collaborateurs qui seront traités injustement dans un compte rendu qui doit rester à l'intérieur de dimensions strictes, fixées à l'avance. 


\section{A. Beltista eiper dunata. Lo statuto dell'utopia nella Repubblica, par Mario Vegetti 107-47.}

Le projet politique de Platon est fondamentalement utopique, dans la mesure où il vise une transformation radicale de la société qui se réaliserait dans le cadre d'une stratégie de rééducation collective. Une entreprise éducative aussi originale ne pouvait être le résultat d'un effort collectif au niveau de la cité, comme c'était le cas pour l'éducation traditionnelle à Athènes. Convaincu que le projet éducatif qu'il proposait ne pouvait avoir de succès que si les bases de la société étaient transformées, Platon propose les réformes radicales énumérées plus haut. Or, comme l'éthique, l'éducation et la politique (au sens strict de gestion du pouvoir législatif de la cité) ne sont pas solidaires, reste posée la question de l'accession au pouvoir, nécessaire afin de rendre possible et efficace ce projet de transformation. Et c'est là que réside le réalisme de Platon, dans la volonté de se doter de l'instrument indispensable pour réaliser ce changement, en fondant l'Académie destinée à fabriquer des súmmakoi et des hetaîroi, sans lesquels l'homme juste est condamné à la solitude que connut Socrate (République VI 496c-d; Lettre VII 325d). Par ailleurs, puisque luimême et les membres de l'Académie devaient penser qu'il était impossible de convaincre le masses de cette réforme, on comprend que Platon ait accepté de prendre des risques pour trouver un tyran qui réalise rapidement cette transformation de la société. Mais, comme on le constate dans la Lettre VII, Platon manquait du réalisme "machiavélique" qui lui eût permis de parvenir à ses fins en ce domaine.

\section{Ba. La prima ondata: il "dramma femminile", par Silvia Campese, 149-95.}

Le titre énigmatique de cette section vient d'une réplique de Socrate: "Aussi convient-il peut-être qu'après avoir mis en scène les hommes et bien déterminé leur rôle, j’y mette les femmes à leur tour." (V 451b-c). En accord avec le principe attribué aux Pythagoriciens: "Entre amis, tout est commun (koina ta philôn)", les femmes auront, dans la cité juste, les mêmes fonctions et les mêmes occupations que les hommes. Cette proposition constituait une remise en cause radicale du statut de la femme en Grèce ancienne, comme l'explique longuement Silvia Campese en se fondant notamment sur les travaux de Nicole Loraux (sans pour autant négliger une masse considérable de littérature secondaire). Pour Platon, l'idée de mettre la femme sur le même pied que l'homme, même en ce qui concerne la guerre, vient du fait que, en dépit de leur différence biologique, la nature de la femme est identique à celle de l'homme, même si cette identité de nature ne va pas sans une certaine supériorité du genre masculin sur le plan de la force physique notamment. Pourquoi, si l'on a deux mains, se borner à n'en utiliser qu'une seule négligeant l'éducation de l'autre. On trouve une interprétation alternative de cette maxime chez Xénophon où la femme est considérée comme la reine de l'oikos, alors que l'homme continue de régner sans partage sur le plan de la cité.

\section{Bb. Nudità, par Silvia Campese, 197-207.}

C'est dans un tel contexte que doit être replacée la question de la nudité des femmes (V 451c-452e), car elle se pose essentiellement en relation avec les exercices physiques. Sur cette question, traitée par d'autres auteurs antiques, Platon reviendra dans les Lois (VIII 832e sq.).

\section{La technê antilogikê tra erizein e dialegesthai, par Fulvia de Luise et Giuseppe Farinetti, 209-31.}

Lorsqu'il cherche à défendre l'idée d'une communauté des fonctions entre l'homme et la femme, Socrate fait une remarque sur les distinctions (V 454a) entre l'antilogikê tekhnê, l' erízein et le dialegesthai. L'antilegein est la technique qui consiste à relever les erreurs logiques et conceptuelles dans les discours de l'adversaire; cette pratique revêt une efficacité redoutable pour ce qui est de la réfutation, notamment parce qu'elle s'interroge sur la rigueur du raisonnement tenu, sur la validité de son point de vue et sur sa cohérence interne. Voilà pourquoi l'antilogie se situe entre l'éristique et l'elenkhos. L'éristique comporte une intention compétitive, qui mène à utiliser tous les types d'expédients logiques pour arriver à vaincre dans la discussion. L'elenkhos, tel du moins que le pratique Socrate en tout cas, se développe dans un esprit d'amitié et de recherche de la vérité, tout en pouvant être difficilement distingué de l'antilogía sur le plan de la procédure mise en œuvre et de la contradiction recherchée. Platon devait donc reconnaître l'utilité de l'antilegein pour affiner la méthode dialectique qui dérive de l'elenkhos.

\section{L'utopia dalla commedia al dialogo platonico, par Anna Beltrametti, 233-56.}

Comme fondateur de la philosophie politique et maître de l'utopie, Socrate continue à être la victime de la politique réelle dans la mesure où, en condamnant les travers des comportements de l'Athènes historique, il déchaîne les rires du public. Dans cette perspective, le terme "comédie" doit être pris en deux sens. Il s'agit de la mise en scène et en cause des vices de la classe politique athénienne par Socrate, et de la réaction que Socrate déclenche chez ceux qui sont les observateurs de ses faits et gestes, lesquels réagissent, en considérant comme ridicules (V 452a sq.) les propositions qu'il vient de faire concernant la communauté des fonctions chez l'homme et chez la femme.

\section{E. La seconda ondata: la comunanza di donne e figli, par Silvia Campese, 257-93.}

La discussion relative à sa première proposition se termine à la pleine satisfaction de Socrate. Il a réussi à montrer la possibilité et l'utilité du fait que les hommes et les femmes accomplissent en commun les mêmes occupations. Mais se profile à l'horizon un seconde problème (V 457c-471c). Il s'agit en effet de reconnaître la pulsion érotique entre les hommes et les femmes qui seront gardiens et de situer cette pulsion dans le cadre d'une proximité sexuelle. Cela dit, il faut se garder de céder au laxisme, car le désir doit obéir à des règles, et cela pour que les produits de l'accouplement soit des agathoi. La communauté des femmes et des enfants transforme la famille particulière en une famille civique qui se trouve illustrée et justifiée par le mythe de l'autochtonie (III 414d-e). 
L'autochtonie fonde la démocratie masculine, car elle dilue la maternité et crée une souche, pure et solidaire, de pères et de fils, la maternité collective impliquant l'anonymat du lien affectif. Mais l'abolition de la sphère du privé produit une conséquence symétrique. Cet anonymat neutralise en effet la notion de père de famille. Sur la scène de la cité les femmes des gardiens sont des homophueis, leurs sœurs politiques. Le mythe de l'autochtonie raconté par Socrate propose donc non seulement une formulation de l'imaginaire patriarcal, mais il introduit un autre imaginaire radicalement nouveau: l'uniformité et la pacification des rôles sexuels dans la cité.

\section{F. La "razza pura", par Mario Vegetti, 295-300.}

L'engendrement des enfants dans la cité constitue évidemment un problème politique, ce qui a amené à parler d'"eugénisme" (V 461a) chez Platon. Platon conjugue un profond pessimisme anthropologique avec un extraordinaire optimisme sur la puissance de l'action politique et de l'éducation. C'est dans ce pessimisme, qu'il ne faut cependant pas dissocier de son optimisme, que s'enracine l'eugénisme platonicien. Pour transformer l'être humain, il faut d'abord prendre des mesures pour constituer le groupe de ceux qui, étant meilleurs, pourront gouverner au nom de toute une cité qui soit finalement une cité d'êtres humains véritables. Dans cette perspective, l'idée est de créer une élite morale et intellectuelle plutôt que d'intervenir sur tel ou tel trait physique.

\section{G. La guerra della kallipolis, par Silvia Gastaldi, 301-34.}

Par la suite, Socrate discute des caractéristiques et des modalités de l'activité guerrière (V 466e-471c). Dans la République, le courage des guerriers sera honoré sur le modèle des honneurs rendus aux guerriers homériques, Homère tout comme Hésiode se voyant ainsi réhabilités sur ce point. En fait, la fonction combattante tournée vers la sauvegarde de la cité trouve sa consécration suprême dans l'attribution d'une "nature démonique" aux soldats tombés courageusement, et mis sous le patronnage de l'Apollon Delphique. Cela dit, Platon réclame que la violence entre les cités grecques soit réglementée. L'idée est d'éviter, dans toutes la mesure du possible, l'affrontement entre Grecs, qui par certains côtés, s'apparente à une guerre civile; les Grecs doivent se montrer solidaires face aux barbares qui sont leurs véritables ennemis.

\section{H. Il regno filosofico, par Mario Vegetti, 335-64.}

En ce qui concerne la troisième réforme susceptible de hâter l'avènement de la cité juste, à savoir l'attribution du pouvoir aux philosophes (V 471c- VI 541b), il semble que Platon, dans la République, adopte deux lignes théoriques. La première fait de la polis le telos de la construction éthico-politique, à l'égard de laquelle la philosophie est un instrument subordonné, même s'il est nécessaire. Dans ce contexte, le pouvoir philosophique évoqué au livre V est la condition de possibilité du gouvernement normal desphulakes / arkhontes décrit dans les livres II à IV, et se trouve confirmé par les analyses du livre VIII. La seconde est une inversion de la première. Le telos devient alors la philosophie, à l'égard de laquelle la cité n'a qu'un rôle instrumental. C'est le thème de la fin du livre VI et de la plus grande partie du livre VII. La tension entre ces deux lignes avaient déjà été remarquée par les pythagoriciens. En fait la ligne de rupture est entre la finalité politique de la philosophie et la finalité philosophique de la cité.

\section{Teoria delle idee e ontologia, par Franco Ferrari, 365-91.}

La discussion que Socrate et Glaucon consacrent à la science et à l'opinion comme à leurs objets respectifs (V 475e6-480a13) intéresse bien évidemment la doctrines des formes et la question de la participation des sensibles à ces formes en 475e9-476d6; on y retrouve les oppositions habituelles, à savoir un/plusieurs, être/paraître, identique/semblable, ce qui est participé/ce qui participe. Il ressort de l'analyse de ce passage que la forme correspond à l'être au sens plein, qu'elle est la référence primaire et qu'elle représente l'unité. Platon insiste moins ici sur l'intelligibilité de la forme que sur la perfection de son identité, qui fonde l'unité, laquelle rend la Forme indépendante de toute variation et lui permet donc de devenir l'objet de l'epistêmê.

\section{Ma. Aristotele discute la Repubblica, par Francesca Calabi, , 421-38}

Il s'agit là d'une analyse de la critique du livre V de la République, qu'on trouve dans le livre II de la Politique (chap. 1-6, 1260b351265a10) et qui pose ces deux questions. Qu'est-ce qui intéresse Aristote chez Platon: quels thèmes, quels passages? 2) Quels genres d'observations Aristote fait-il à Platon? Aristote ne parle pas seulement de l'impossibilité de la cité de la République, mais aussi et avant tout de son caractère irréalisable.

\section{Mb. La critica aristotelica alla Repubblica nel secondo libro della Politica, il Timeo et le Leggi, par Mario Vegetti, 439-52.}

La critique aristotéliciennne dans la Politique refléterait les discussions au sein de l'Académie, et auraient laissé des traces dans deux dialogues ultérieurs, à savoir le Timéeet les Lois.

\section{N. La kallipolis di Rousseau, par Fulvia de Luise, 453-96.}

Analyse des prises de positions de Rousseau face aux réformes proposées dans le livre $\mathrm{V}$ de la République. Rousseau est d'accord avec les deux premières. Il interprète la notion du philosophe-roi, en termes de possibilité pour le peuple de progresser dans la perfection sur le plan de la volonté générale. Il admet aussi la communauté des biens interprétée en terme d'échange symbolique, garantie de la liberté. Mais Rousseau exprime son désaccord total sur la question de la place et du rôle de la femme dans la société. 


\section{O. Il confronto di Marx con Platone (attraverso Hegel), par Guiseppe Farinetti, 497-559.}

Dans leur ingénuité les Grecs représentent le stade infantile de l'humanité, car ils considérent l'homme comme le but de toute activité sociale, y compris de la production des biens. Le monde moderne, fondé exclusivement sur la valeur d'échange, propose une communauté centrée sur la production de bien en y subordonnant l'homme: pour obtenir sa liberté, l'homme doit alors renoncer à toute notion de propriété. Telle est distinction avec le marxisme, qui se fait jour sur la question de l'interdiction faite par Platon aux gardiens et aux philosophes de posséder le moindre bien.

À l'occasion de la traduction et du commentaire du cinquième livre de la République, je renouvelle les éloges que j'avais faits lors de la parution des trois premiers volumes de cette entreprise. Comme on peut le constater, ce travail renouvelle notre approche de la République de Platon, et présente l'intérêt de donner l'exemple de l'application de méthode très différente de celle mise en œuvre dans les commentaires les plus connus à l'époque actuelle. Cette méthode s'inscrit par ailleurs dans un mouvement de réflexion sur la culture et l'histoire de la Grèce ancienne par un certain nombre de chercheurs contemporains. Mais cette méthode suscite une critique qui tient aux conditions mêmes de son application. L'ensemble des participants met en effet le projet de Platon sur le même plan que tous les projets politiques qui ont été formulés dans le cours des temps, celui de la réalité matérielle. Mais chez Platon, il faut aussi prendre en compte la distinction entre l'âme et le corps, entre l'intelligible et le sensible.

La distinction intelligible / sensible est excellemment traitée ici par Franco Ferrari et elle reviendra dans les livres VI et VII notamment. Mais l'opposition âme / corps n'est pas vraiment prise en compte, et cela pose des problèmes inextricables. La question essentielle est la suivante: qu'est-ce qu'un être humain? La réponse habituelle, un corps animé insiste sur le corps, ce qui correspond à la pratique sociale qui organise tout en fonction des besoins du corps. Or, la réponse de Platon est paradoxale. Pour lui, un être humain, c'est avant tout une âme. À partir de là, la distinction fonctionnelle entre l'homme et la femme, fondée sur des caractéristiques biologiques - être pourvu(e) d'un sexe féminin ou d'un sexe masculin - s'effondre; seule subsiste une différence de degré, le corps de l'homme étant plus puissant qu'un corps de femme. Or, si la distinction homme / femme ne tient plus lorsque l'on considère les choses du point de vue de l'âme, il n'y a plus aucune raison pour écarter la femme du métier des armes. Il s'ensuit que, si l'objectif de la relation sexuelle considérée comme exclusive n'est plus la transmission de son capital génétique et foncier, la famille n'a plus aucun sens; dans cette perspective, 1'"eugénisme" intéresse avant tout l'âme, et voit son centre de gravité déplacé. Et si c'est le bien de l'âme qui compte, le pouvoir ne peut plus venir de la richesse ou de la force, mais doit être dévolu à ceux qui font de l'activité de l'âme leur activité exclusive, à savoir les philosophes. Nous retrouvons de ce fait les trois conditions de possibilité de la cité juste, considérées cette fois non plus sous le point de vue de l'âme, mais sous celui du corps.

Dans la République, Platon cherche bien à décrire la naissance et la constitution d'une cité réelle, communauté de corps humains animés sur un territoire donné, mais il considère que le soin du corps est moins important que celui de l'âme auquel doit s'intéresser en premier lieu le fondateur de cité. Voilà pourquoi, je le répète, la Républiquese présente avant tout comme une psychologie politique.

Cela critique qui se voudrait l'amorce d'une discussion ne remet pas en cause l'originalité et la qualité du travail entrepris par Mario Vegetti et son équipe. J'espère que les prochains volumes seront rapidement publiés.

\section{LUC BRISSON}

Centre Nationale de la Recherche Scientifique, Paris.

France 